\title{
Characterization Of Pseudo-Range Multipath Errors In An Urban Environment
}

\author{
Eustachio Roberto Matera, Axel Garcia-Pena, Olivier Julien, Bertrand Ekambi
}

Ecole Nationale de l'Aviation Civile (ENAC), ABBIA GNSS Technologies, Toulouse, France

\section{BIOGRAPHIES}

Eustachio Roberto Matera is currently a $\mathrm{PhD}$ student at ENAC. He has obtained telecommunication engineer diploma in 2014 and a Master of Science in wireless communication in 2016 at Politecnico di Torino, Italy. He is currently studying signal processing algorithms and integrity monitoring techniques adapted to the navigation in urban areas. His thesis is funded by ABBIA GNSS Technologies.

Axel Garcia Pena is a researcher/lecturer with the SIGnal processing and NAVigation (SIGNAV) research group of the TELECOM lab of ENAC (French Civil Aviation University), Toulouse, France. His research interests are GNSS navigation message demodulation, optimization and design, GNSS receiver design and GNSS satellite payload. He received his double engineer degree in 2006 in digital communications from SUPAERO and UPC, and his $\mathrm{PhD}$ in 2010 from the Department of Mathematics, Computer Science and Telecommunications of the INPT (Polytechnic National Institute of Toulouse), France.

Olivier Julien is the head of the SIGnal processing and NAVigation (SIGNAV) research group of the TELECOM lab of ENAC (French Civil Aviation University), Toulouse, France. His research interests are GNSS receiver design, GNSS multipath and interference mitigation, and interoperability. He received his engineer degree in 2001 in digital communications from ENAC and his PhD in 2005 from the Department of Geomatics Engineering of the University of Calgary, Canada.

Bertrand EKAMBI graduated by a Master in Mathematical Engineering in 1999. Since 2000, he is involved in the main European GNSS projects: EGNOS and GALILEO. He is the founder manager of ABBIA GNSS Technologies, a French SME working on Space Industry, based in Toulouse, France.

\begin{abstract}
An increasing number of new applications require an accurate positioning even in urban environments; however, in such environments, especially in urban canyons, GNSS positioning is challenged to meet the applications' demanded accuracy. In fact, in order to obtain an optimal and reliable position estimate using GNSS, it is necessary to have an accurate model of the pseudorange and pseudorange rate error terms' distributions.
\end{abstract}

This work focuses thus its attention on the statistical characterization of the pseudorange measurements' multipath error component by proposing a methodology to obtain such characterization: isolation of the multipath error component from the use of a reference station, to eliminate ionospheric error terms, and from a filtering process, to eliminate receiver clock bias. The methodology is applied to real measurements obtained from a data campaign conducted in Toulouse urban area with a u-Blox receiver with its antenna mounted on the roof of a car. The characterization process is conducted from pseudorange measurements of GPS constellation taken in the L1 band.

In order to obtain a better characterization, the pseudorange measurements are classified by the signal $C / N_{0}$ and by the elevation angle between the satellites and the receiver, which are common signal characteristics influencing the multipath error component impact on the pseudorange measurement. The performance assessment of each parameter in terms of signal reception conditions classification between LOS and NLOS has determined the upper hand of the $C / N_{0}$ parameter.

\section{INTRODUCTION}

Nowadays, navigation systems integrating at least inertial measurement unit (IMU) and Global Navigation Satellite System (GNSS) signal processing units are becoming the fundamental baseline platform for mass-market user devices. Such platforms aim at combining reasonably lowcost hardware with the provision of the highest possible positioning accuracy, availability and reliability. However, the hybrid system will still rely on GNSS measurements alone for correcting the 
IMU increasing-in-time bias errors. Therefore, in order to obtain an optimal and reliable position estimate, it is necessary to have access to accurate GNSS pseudorange measurements even in difficult environments, or at least to a precise characterization /assessment of the GNSS pseudorange measurements.

Urban environments present a significant challenge for modern Global Navigation Satellite Systems (GNSS). GNSS were originally designed to work in open areas, where the satellites are always in direct line-of-sight (LOS) from the receiver. However, in urban environments, the satellite signals reception conditions are much harsher: surrounding obstacles reflect/diffract the satellites transmitted signals, creating the multipath phenomenon, or even block the LOS signal, creating non-line-of-sight (NLOS) received satellite signal conditions. As a result, the position estimation will have larger errors than in open sky conditions. Nevertheless, a good characterization of these effects could help with the loss of positioning performance (such as accuracy or integrity) with respect to the error in open-sky environments.

The multipath effect on GNSS receivers has been theoretically studied in the literature with a main focus on fixed position test conditions. These studies have analysed the effects of multipath on the pseudorange measurements [1][2]. Moreover, multiple studies based on simulations have also been conducted to address the multipath impact on the code phase and the carrier phase measurements [3][4].

In this paper, the general goal is thus to present a methodology to characterize the multipath error impact on the pseudorange measurements being observed by a GPS receiver in the L1 frequency band on-board a mobile platform traversing a densely built area.

In this paper, the methodology proposed to characterize the multipath error component impact on the pseudorange measurement consists of the following 2 steps:

1- A multipath error component isolation method from the L1 band pseudorange measurement is applied [9].

2- A multipath error characterization process is conducted: the probability density function is estimated and classified as a function of the satellite $C / N_{0}$ and the elevation angle between the satellite and the receiver.

Finally, a real application example of the proposed methodology is conducted based on a data measurement campaign performed in Toulouse urban area, and additionally, from these results, the LOS and NLOS classification performance of each parameter has been assessed

The paper is organized as follows. Section 2 describes the multipath error isolation method. Section 3 describes the multipath error component characterization process. Section 4 describes the experimental setup used to conduct the signal test campaign as well as the vehicle trajectory inside Toulouse urban area. The results obtained are presented and analysed in Section 5. Finally, a discussion on the methodology used is made and conclusions are given about the article's main results regarding their exploitation for GNSS vehicle applications.

\section{MULTIPATH ERROR COMPONENT ISOLATION METHOD}

This section presents the theoretical fundamentals on which the multipath error isolation method is based.

\subsection{Multipath isolation: fundamental idea}

The code pseudorange measurement obtained by a user receiver from satellite $i$ at a given instant $t$ can be accurately modelled as [6]:

$$
\begin{gathered}
P^{i}(t)=R^{i}(t)+c\left(\Delta t_{u}(t)-\Delta t_{s v}^{i}(t)\right)+ \\
+c \Delta t_{i o n}^{i}(t)+c \Delta t_{t r o}^{i}(t)+M P_{u}^{i}(t)+b h w_{u}(t)+ \\
+b h w_{s v}^{i}(t)+\eta_{u}^{i}(t)
\end{gathered}
$$

where

- $\quad R^{i}(t)$ is satellite-to-receiver range at time $t$ $\sqrt{\left(x_{s v}^{i}(t)-x(t)\right)^{2}+\left(y_{s v}^{i}(t)-y(t)\right)^{2}+\left(z_{s v}^{i}(t)-z(t)\right)^{2}}$ it is the effective range between the satellite and the receiver at epoch $t$, which could be used for positioning purpose.

- $\quad(x, y, z)$ are the receiver true coordinates

- $\left(x_{s v}^{i}, y_{s v}^{i}, z_{s v}^{i}\right)$ are the satellite $i$ true coordinates for the satellite

- $\quad c$ is the speed of light

- $\Delta t_{u}$ is the receiver clock bias

- $\Delta t_{s v}^{i}$ is the satellite $i$ clock bias

- $\Delta t_{\text {ion }}^{i}$ is the ionospheric delay from satellite $i$ measurement

- $\Delta t_{\text {tro }}^{i}$ is the atmospheric delay from satellite $i$ measurement

- $\quad M P_{u}^{i}$ is the code multipath error component of the user from satellite $i$ measurement

- $\quad b h w_{u}$ is the receiver hardware bias

- $\quad b h w_{s v}^{i}$ is the satellite $i$ hardware bias

- $\quad \eta_{u}^{i}$ is the random measurement noise of the user from satellite $i$ measurement

In order to isolate/estimate the multipath error component, $M P^{i}(t)$, the other terms from the model proposed in equation 2.1 must be removed. 
The main idea of the proposed method consists in:

- removing all geometry elements from the pseudoranges through a combination of differencing with a reference station and use of the known location of the user

- $\quad$ estimating and removing the clock terms.

\subsection{Isolation of Multipath and Clock Terms}

The first step of the multipath error isolation is to obtain a pseudorange residual which contains only the pseudorange error terms. To obtain such residual, the true receiver-to-satellite range term must be removed.

\subsection{1 "Range-Free" Measurements}

In the present case, it is assumed that the exact location of the user and reference station antennas are known. This is very easy for the reference station, while the data collection that will be detailed later on used a high precision system to provide the accurate user location even in an urban environment.

For a receiver, at given time $t_{0}$ and in a known location, $\left(x_{0}=x\left(t_{0}\right), y_{0}=y\left(t_{0}\right), z_{0}=z\left(t_{0}\right)\right)$, it is possible to precisely estimate the true receiver-tosatellite $i$ range as in 2-2:

$$
\sqrt{\left(x_{e s t}^{i}(t)-x_{0}\right)^{2}+\left(y_{e s t}^{i}(t)-y_{0}\right)^{2}+\left(z_{e s t}^{i}(t)-z_{0}\right)^{2}}
$$

where the satellite position, $\left(x_{\text {est }}^{i}(t), y_{\text {est }}^{i}(t), z_{\text {est }}^{i}(t)\right)$, is estimated from the ephemeris file broadcasted by the satellite.

The "range-free" user pseudorange residuals (per satellite) can thus determined by differencing the estimated true receiver-to-satellite range (also called effective range), equation 2-2Erreur ! Source du renvoi introuvable., and the pseudorange measurement, equation 2-1. The mathematical expression is given in 2-3Erreur ! Source du renvoi introuvable.,

$$
\begin{gathered}
\Delta_{R X}^{\mathrm{i}}(t)=P^{i}(t)-R_{0}^{i}(t) \\
=c\left(\Delta t_{U}(t)+\Delta t_{s v}(t)\right)+E_{u}^{i}(t)+c \Delta t_{i o n}^{i}(t)+ \\
+c \Delta t_{t r o}^{i}(t)+M P_{u}^{i}(t)+b h w_{u}(t)+b h w_{s v}^{i}(t)+ \\
+\eta_{u}^{i}(t)
\end{gathered}
$$

where $E_{u}^{i}$ is the residual error projected on the pseudorange domain due to the satellite $i$ position estimation error. The same can be done to obtain "range-free" reference station pseudorange residuals, 2-4:

$$
\begin{gathered}
\Delta_{\text {sta }}^{\mathrm{i}}(t)= \\
=c\left(\Delta t_{\text {sta }}(t)+\Delta t_{\text {sv }}(t)\right)+E_{\text {sta }}^{i}(t)+c \Delta t_{\text {ion }}^{i}(t)+ \\
+c \Delta t_{\text {tro }}^{i}(t)+M P_{\text {sta }}^{i}(t)+b h w_{\text {sta }}(t)+b h w_{\text {sv }}^{i}(t)+ \\
+\eta_{\text {sta }}^{i}(t)
\end{gathered}
$$

where

- $\Delta t_{\text {sta }}$ is the reference station clock bias

- $\quad E_{\text {sta }}^{i}$ is the reference station pseudorange residual error projected on the pseudorange domain due to the satellite $i$ position estimation error

- $M P_{\text {sta }}^{i}$ is the code multipath error component of the reference station from satellite $i$ measurement

- $b h w_{\text {sta }}$ is the reference station receiver hardware bias

- $\quad \eta_{\text {sta }}^{i}$ is the random measurement noise of the reference station from satellite $i$ measurement

\subsubsection{Pseudorange residual difference}

The second step of the proposed method consists in removing the impairments from the vehicle receiver pseudorange residual which are common to the reference station ones. The removal is simply achieved by differencing the user "range free" measurement $\Delta_{R X}^{\mathrm{i}}(t)$ from the "range free" reference station pseudorange residual $\Delta_{s t a}^{\mathrm{i}}(t)$, equation 2-5.

$$
\begin{gathered}
\epsilon_{P S R}^{i}(t)=\Delta_{R X}^{\mathrm{i}}(t)-\Delta_{s t a}^{\mathrm{i}}(t)= \\
=c\left(\Delta t_{u}(t)-\Delta t_{\text {sta }}(t)\right)+E_{u}^{i}(t)-E_{\text {sta }}^{i}(t)+ \\
+M P_{u}^{i}(t)-M P_{s t a}^{i}(t)+b h w_{u}(t)-b h w_{\text {sta }}(t)+ \\
+\eta_{u}^{i}(t)-\eta_{\text {sta }}^{i}(t)
\end{gathered}
$$

The resulting term, $\epsilon_{P S R}^{i}(t)$, is denoted as the pseudorange residual difference and is dominated by five factors:

a. the vehicle-reference station receiver clock difference, $\left(\Delta t_{u}(t)-\Delta t_{s t a}(t)\right)$;

b. a residual error due to the satellite position estimation error, induced by the broadcast ephemeris, projected on the pseudorange domain, $E_{u}^{i}(t)-E_{s t a}^{i}(t)$;

c. the user receiver multipath error component, $M P_{u}^{i}(t)$ and the reference station multipath error component, $M P_{s t a}^{i}(t)$

d. the receivers' bias hardware term, $b h w_{u}(t)-b h w_{s t a}(t)$

e. the user receiver noise, $\eta_{u}^{i}(t)$ and the reference station receiver noise, $\eta_{\text {sta }}^{i}(t)$.

Let us assume that:

- the multipath error component experienced by the test receiver is much greater than those experienced by the reference receiver (due to the signal reception environment and the receiver quality), the pseudo-range 
residual difference can be considered to be dominated by the receiver residual errors.

- the residual ephemeris errors difference, $E_{u}(t)-E_{\text {sta }}(t)$, are negligible in front of the multipath error.

- the receiver and satellite hardware bias slowly vary during the measurement campaign,

- the vehicle-reference station receiver clock difference, $\left(\Delta t_{u}(t)-\Delta t_{s t a}(t)\right)$, and the receivers' bias hardware term, $b h w_{u}(t)-$ $b h w_{\text {sta }}(t)$ are estimated together as a unique term called clock bias term, $\Delta t_{u}^{h}$.

Under these assumptions, the differential measurement can be simplified into (2-6):

$$
\epsilon_{P S R}^{i}(t) \approx c \Delta t_{u}^{h}(t)+M P_{u}^{i}(t)+\eta_{u}^{i}(t)-\eta_{s t a}^{i}(t)
$$

where $M P_{u}^{i}$ now includes the reference multipath as well as the residual atmospheric errors and residual ephemeris errors.

Finally, the following assumptions/conditions must be met in order to successfully apply the proposed method:

- The position of the vehicle receiver antenna is known with a decimetre-level accuracy during the whole trajectory.

- The position of the reference station receiver antenna is known.

- All the reference station measurements are supposed to have a multipath error component negligible with respect to the multipath error component of the vehicle receiver pseudorange measurement.

- The distance between the vehicle and the reference station is sufficiently small to assume that they are affected by the same atmospheric impairments.

\subsubsection{Multipath error component isolation from pseudorange residual difference method}

The last step of the proposed multipath error component isolation method consists in isolating the multipath error component from the clock bias term, $c \Delta t_{u}^{h}(t)$. The isolation is conducted by:

1- Estimating $c \Delta t_{u}^{h}(t)$ from the pseudorange residual difference terms

2- Removing/subtracting the estimated clock bias term, $c \widehat{\Delta t}_{u}^{h}$, from each pseudorange residual difference, $\epsilon_{P S R}^{i}$, to estimate each individual $\widehat{M P}^{i}(t)$.
Erreur ! Source du renvoi introuvable. presents the block diagram of this method.

A very important remark which must be made about the proposed method to estimate the multipath error component is that, as the reader will observe, this method does not differentiate between the multipath error, $M P_{u}^{i}(t)$, and the thermal noise, $\eta_{u}^{i}(t)-\eta_{\text {sta }}^{i}(t)$, components. In fact, the estimate provided by this method will also contain the thermal noise components in addition to the multipath error component. Therefore, the final estimated term should be analysed and exploited by keeping in mind such fact. Moreover, the final estimate should thus be noted as $\widehat{M P N}^{i}(t)$ in order to indicate the inclusion of the thermal noise terms.

Two fundamental facts are the basis of clock bias term, $c \Delta t_{u}^{h}(t)$, estimation process (step 1 of the multipath error component isolation from pseudorange residual difference method). First, the estimation process exploits the fact that the clock bias term is considered to be the component with the lowest frequency spectrum among the pseudorange residual difference terms. Therefore, one possible way to estimate $\Delta t_{u}^{h}(t)$ is to apply a lowpass frequency filter to the any pseudorange residual difference term [8]. Note that the selected cut-off frequency must be high enough not to filter out the clock bias term but low enough to filter out, as much as possible, the averaged noise and multipath terms. The selected cut-off frequency value can be found in the experimental parameters list in section Erreur ! Source du renvoi introuvable.

Second, the clock bias term, $\Delta t_{u}^{h}(t)$, is common to all pseudorange residual difference terms belonging to the same time epoch whereas the multipath error and thermal noise components are different. Therefore, in order to reduce the impact of the receiver noise and multipath error components on the clock bias term estimate, the different satellite pseudorange residual differences per time epoch can be averaged together prior to the filtering process. However, this averaging process has to be performed with care when considering an urban scenario since some measurements might be strongly corrupted (for instance NLOS situations). Therefore, the averaging operation is performed only on a pseudorange residual subset where such a chosen subset is characterized by a high level of $C / N_{0}$. More specifically, only satellites with a $C / N_{0}$ higher than a certain threshold will be used for the clock bias term estimation process. 
Equation 2-7 shows the averaging process, obtaining, as a result, the averaged pseudorange residual difference, $\varepsilon_{P S R}(t)$,

$$
\begin{gathered}
\varepsilon_{P S R}(t)=\frac{\sum_{i=1}^{N_{\text {sat }}(t)} \epsilon_{P S R}^{i}(t)}{N_{\text {sat }}(t)}= \\
=c \Delta t_{u}^{h}(t)+\frac{\sum_{i=1}^{N_{\text {sat }}(t)}\left(M P_{u}^{i}(t)+\eta_{u}^{i}(t)-\eta_{\text {sta }}^{i}(t)\right)}{N_{\text {sat }}(t)}
\end{gathered}
$$

where $N_{\text {sat }}(t)$ is the number of satellites over the threshold at given time epoch $t$.

Equation (Erreur ! Source du renvoi introuvable.) presents the mathematical expressions of the estimated clock bias term, $c \widehat{\Delta t}_{u}^{h}(t)$, and multipath error and thermal noise components term, $\widehat{M P N}^{i}(t)$.

$$
\begin{gathered}
c \widehat{\Delta t}_{u}^{h}(t)=L P F\left\{\varepsilon_{P S R}(t)\right\} \\
\widehat{M P N}^{i}(t)=\epsilon_{P S R}^{i}(t)-c \widehat{\Delta t}_{u}(t)
\end{gathered}
$$

where $L P F\{\ldots\}$ is the low pass filtering operation.

Finally, in order to keep a compact notation, the remaining part of this article will continue to refer to the estimate $\widehat{M P N}^{i}(t)$ as the multipath error component estimate. Nevertheless, the reader must not forget that this term also contains the thermal noise components.

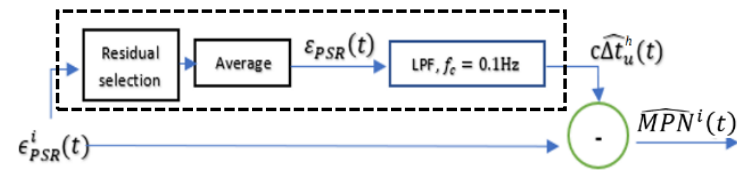

Figure 1 - Schematic of pseudo-range multipath error isolation from the residual containing multipath error, clock bias term and noise

\section{MULTIPATH ERROR COMPONENT CHARACTERIZATION}

Once the multipath error component, $\widehat{M P N}^{i}$, is isolated, the multipath characterization process can be applied in order to obtain a mathematical model, such as a random process, which defines the multipath error component statistical behaviour.

Moreover, in order to obtain a finer characterization or modelling of the multipath error component, first, the multipath error component values are divided into different groups, where each group represents a specific key received satellite signal reception condition, and second, the characterization process is conducted per group. By doing so, specific models more representative of a given situation are obtained.
Two types of classification are considered in this work.

First, all the received satellites signals can be classified depending on the signal $C / N_{0}$ value. Moreover, the $C / N_{0}$ is an indicator used in the receiver position estimation which determines the quality of the received signal. Therefore, it is a good indicator of the signal reception quality:

- low $C / N_{0}$ means higher probability to get errors in the pseudorange measurement domain; strong multipath error component impact

- $\quad$ high $C / N_{0}$ means that the signal is received with a higher level of carrier power wrt the noise; a priori weak multipath error component impact.

Second, received satellite signals can be also classified depending on the signal elevation angle value. The elevation angle could be a good indicator of the multipath error component impact with respect to the LOS signal in the pseudorange measurement domain in the case of urban environment:

- low elevation angle means higher probability to get errors in the pseudorange measurement domain due to higher probability of the satellite to be obstructed by obstacles; strong multipath error component impact and high probability to be a NLOS signal.

- $\quad$ high elevation angle means that the satellite is higher, with low probability to be obstructed by obstacles; weak multipath error component impact and low probability to be a NLOS signal.

In the following, the measurements will be characterized as a function of the $\mathrm{C} / \mathrm{N}_{0}$ and satellite elevation.

\section{EQUIPMENT DESCRIPTION}

The equipment used during the data collection campaign consists of the following devices:

a. U-Blox M8T: GPS receiver.

b. Novatel \& Span GPS/GLONASS receiver.

c. CNES Toulouse reference station receiver

d. Laptop used for the experiment

e. ENAC test vehicle used to do the experimental data campaign.

The role of the uBlox M8T receiver in the experiment is to be the low-cost "mass-market" receiver working in the $\mathrm{L} 1$ frequency band which collects the data to be analysed. 
The role of the NovAtel SPAN receiver is to obtain a very precise trajectory of the car during the data collection campaign which will be used as the true position of the receiver at any instant of time. Moreover, the SPAN receiver is also responsible for providing the vehicle heading information which can be extrapolated to the test receiver antenna using the known level-arm between IMU and the antenna.

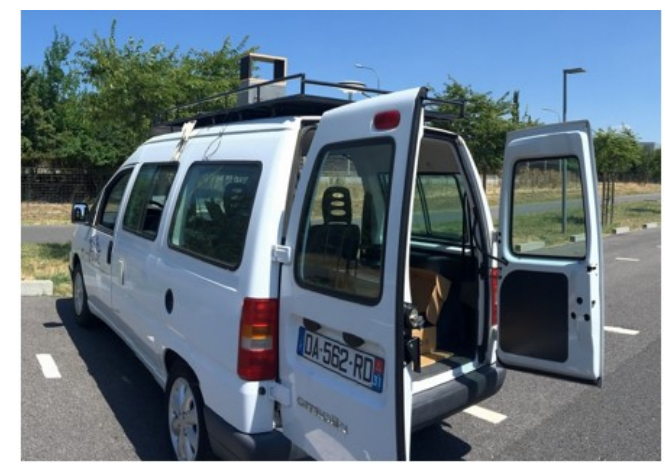

Figure $2 a$ - Picture of the vehicle used for the experiment

The SPAN receiver accuracy is at the decimetrelevel or better [9].

The reference station used in the experiment is the one located in the Toulouse site of the Centre National d'Etudes Spatiales (CNES). Figure 2 Erreur ! Source du renvoi introuvable.a shows the test vehicle. Inside the van a small laboratory allows to work with the laptops and take the data campaign under control.

\subsection{Set-up description}

In Figure 2Erreur! Source du renvoi introuvable.b, a picture of the equipment set-up mounted on the roof of the vehicle is presented. The U-Blox M8T and NovAtel antennas are installed on the roof of the ENAC test vehicle. The Novatel GNSS module, the IMU sensors, the consumergrade GNSS receiver as well as the computers, which records the GNSS data, are inside the vehicle. A scheme of the physical set-up of these elements is shown in Figure 4a and Figure 4b.

The data from uBlox receiver and NovAtel SPAN receiver are synchronously collected.

The Novatel \& Span collected information is synchronized with the U-Blox data by applying a GPS timestamp to the information. The SPAN receiver uses its internal memory to save the data measurements, as seen in Figure 4b, which means that it is not directly connected to the laptop: the synchronization between the U-Blox, and NovAtel will thus be achieved in the post-processing stage

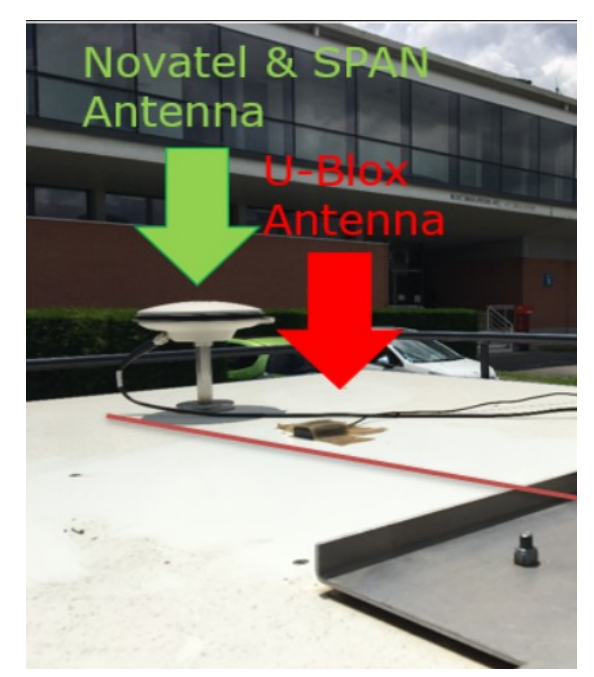

Figure $2 b$-Rooftop of the vehicle, showing the $U$ Blox and NovAtel antennas

\subsection{Trajectory}

The trajectory followed in Toulouse urban area is divided in three different sections, ordered in the chronologically sequence as shown in Figure 3.

The selected location for the data campaign was Toulouse urban area. The location was chosen in order to have a representation of different types of obstacles and different LOS/NLOS scenarios:

- $\quad$ the city centre, with large and small streets around tall buildings and areas including bunch of trees;

- the suburbs; large and small streets in presence of small buildings;

- $\quad$ open areas close to the River Garonne.

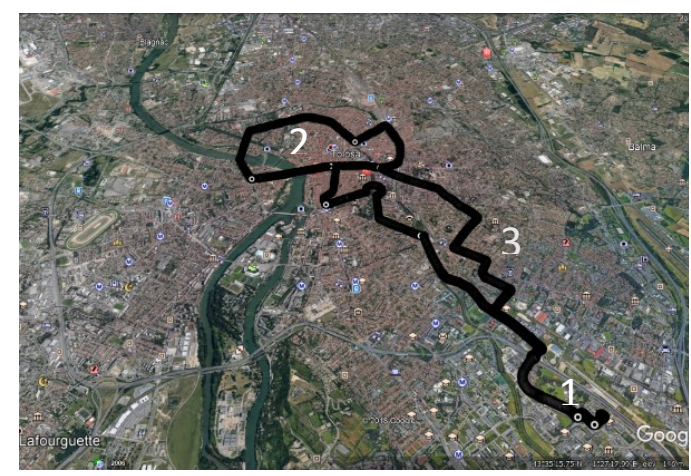

Figure 3 - Trajectory of the data collection experiment. 

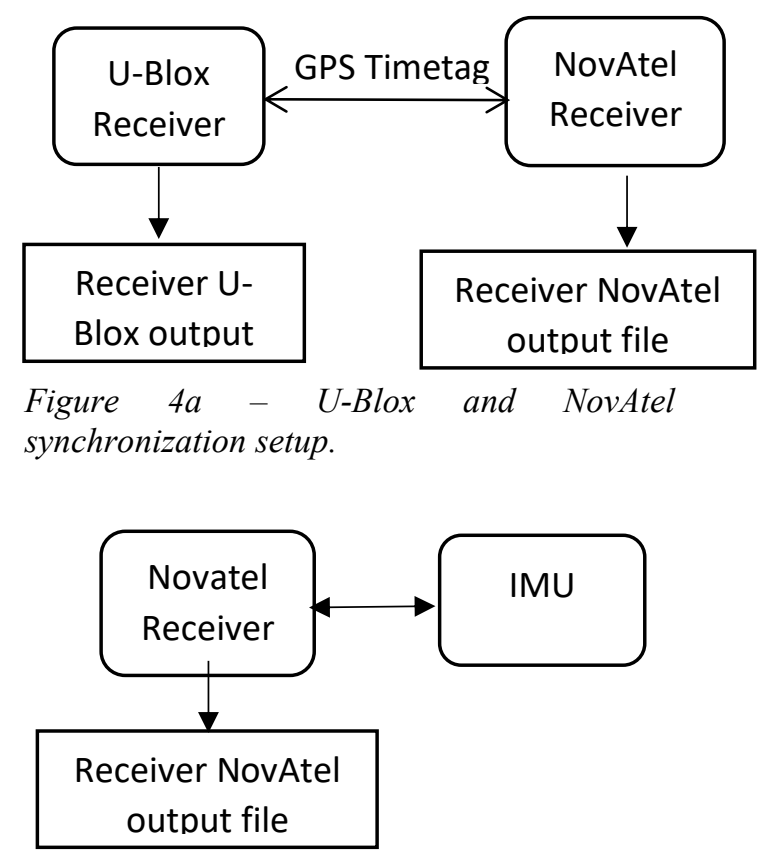

Figure $4 b-$ NovAtel setup. GNSS receiver and IMU work simultaneously.

\section{RESULTS}

The section presents the experimental results. The first two sections include the data campaign and the multipath isolation parameters. The other sections present the results of the multipath isolation. In 5.4the multipath error model characterized by $C \backslash N_{0}$ is presented. In 5.5 the multipath error model characterized by elevation angle is presented. Finally, section 5.6 provides a preliminary comparison between the LOS and NLOS classification performance between the two previous selected signal/scenario parameters.

\subsection{Data campaign parameters}

The following parameters were used to configure the data campaign equipment:

- The u-Blox receiver works in the L1 band, centered at $1575.42 \mathrm{MHz}$.

- The u-Blox's output data frequency is $1 \mathrm{~Hz}$.

- The evaluated constellation is GPS.

- The Novatel SPAN output data frequency is $5 \mathrm{~Hz}$.

- The data collection's duration is 7926 seconds, roughly 2 hours and 20 minutes.

\subsection{Multipath isolation parameters}

Concerning the receiver clock bias estimation during the application of the multipath error component isolation method, the chosen parameters are:
- $C / N_{0}$ threshold set to $35 \mathrm{~dB}-\mathrm{Hz}$ for the receiver clock bias estimation method (see section 2.2.3).

- $\quad$ Selected filter: first order Butterworth filter with cutting frequency equal to $0.1 \mathrm{~Hz}$ [8].

\subsection{Expected behaviour of the estimated multipath error component depending on LOS and NLOS signal reception conditions}

The identification of LOS and NLOS received signals is very important in order to increase the accuracy and the reliability of the final PVT solution. However, such classification is not easily done from the signal/scenario parameters which a low-cost receiver could have access to. Therefore, in order to be able to obtain a preliminary classification or influence of LOS and NLOS conditions on the multipath error component pdf as a function of different accessible signal/scenario parameters (see section 3 ), the results obtained in this section will be analysed depending on the expected behaviour of the multipath error component depending on LOS and NLOS signal reception conditions. In fact, note that the expected multipath error component pdf of a LOS satellite is different from the expected pdf of a NLOS satellite.

important to note that. On one hand, , the estimated multipath error component plus the remaining noise component pdf of a LOS satellite should theoretically be close to a centred Gaussian distribution: since the combination of the LOS signal plus the multipath component can create either a positive or negative error and the thermal noise is centred Gaussian distributed, the expected pdf is also a centred Gaussian distribution but with, probably, a different standard deviation.

On the other hand, the absence of LOS signal should always introduce a positive bias in the pseudorange measurement: the receiver tracks the NLOS signal(s). Therefore, when taking into account the thermal noise component terms, the resulting multipath error component distribution should tend to have a very heavy positive tail.

\subsection{Multipath error characterization with respect to $\mathrm{C} / \mathrm{N}_{0}$ classification}

The list of results obtained with respect to the GPS constellation is presented next. Some of the results are commented first; then, the whole multipath error model is presented.

Figure 5 corresponds to the multipath error component pdf in the $45-50 \mathrm{~dB}-\mathrm{Hz} \mathrm{C} \backslash \mathrm{N}_{0}$ range. The multipath error component has a symmetric Gaussian shape centred in 0 and a standard deviation 
of 0.83 meters. The pdf symmetry implies that the multipath error is mainly generated from LOS signals measurements as stated in section 5.3.

Figure 6 corresponds to the multipath error component pdf in the $35-40 \mathrm{~dB}-\mathrm{Hz} \mathrm{C} \backslash \mathrm{N}_{0}$ range. The pdf seems to be a Non-Gaussian distribution. It can be seen that the total pdf is slightly non-symmetrical: the pdf's values corresponding to the positive multipath errors seem to be higher than the negative part. This phenomenon is probably due to the presence of signals received in NLOS conditions that result in positive biases as stated in section 5.3. The presence of these biases means that there begins to be a non-negligible presence of multipath errors components generated by NLOS satellites.

The effect of NLOS situations can be clearly seen in Figure 7, for the pdf in $25-30 \mathrm{~dB}-\mathrm{Hz} \mathrm{C} \backslash \mathrm{N}_{0}$ range. In this picture, the probability density function peak is located at around 3 meters. The mean is located at 11.48 meters. As well as before, the pdf positive error part is higher than the negative error part. The multipath error component seems thus to be dominated by NLOS satellites.

The last case, the pdf in the $15-20 \mathrm{~dB}-\mathrm{Hz} \mathrm{C} \backslash \mathrm{N}_{0}$ range, is presented in Figure 8. The magnitude of the multipath error component value increases dramatically with the respect to the other cases. The pdf dispersion is larger, and it seems highly biased in the positive part. The multipath error component is clearly dominated by NLOS satellites.

Table lErreur! Source du renvoi introuvable. contains the pdf's mean, standard deviation and the main peak's value for each different pdf characterized by a different $C / N_{0}$ range. From this table, it can be observed that from range 30-35 dB$\mathrm{Hz}$ to lower values, the main peak has a significant difference with respect to the mean value. Moreover, the mean is far from being equal to 0 . Therefore, it can be observed that from the $30-35 \mathrm{~dB}-\mathrm{Hz}$ range, a significant number of NLOS signals are received.

\begin{tabular}{|c|c|c|c|}
\hline $\begin{array}{c}\boldsymbol{C} / \mathbf{N}_{\mathbf{0}} \\
\text { range } \\
\text { (dB-Hz) }\end{array}$ & $\begin{array}{c}\text { Main } \\
\text { Peak (m) }\end{array}$ & $\begin{array}{c}\text { Mean } \\
(\mathbf{m})\end{array}$ & $\begin{array}{c}\text { Standard } \\
\text { deviation } \\
(\mathbf{m})\end{array}$ \\
\hline $0-5$ & No value & No value & No value \\
\hline $5-10$ & 19.6819 & 56.6726 & 72.7046 \\
\hline $10-15$ & 15.1254 & 40.6000 & 47.5482 \\
\hline $15-20$ & 11.5025 & 29.7853 & 33.7863 \\
\hline $20-25$ & 7.7986 & 19.9413 & 22.2415 \\
\hline $25-30$ & 2.0481 & 11.4885 & 16.6950 \\
\hline $30-35$ & -0.0447 & 4.9316 & 11.5438 \\
\hline $35-40$ & 0.3519 & 0.9163 & 5.1890 \\
\hline $40-45$ & 0.1219 & 0.2632 & 1.7252 \\
\hline $45-50$ & 0.0052 & 0.0581 & 0.8290 \\
\hline $50-55$ & -0.0181 & -0.1097 & 0.7153 \\
\hline
\end{tabular}

Table 1 - Multipath error component pdf's characteristics per different $C / N_{0}$ range.

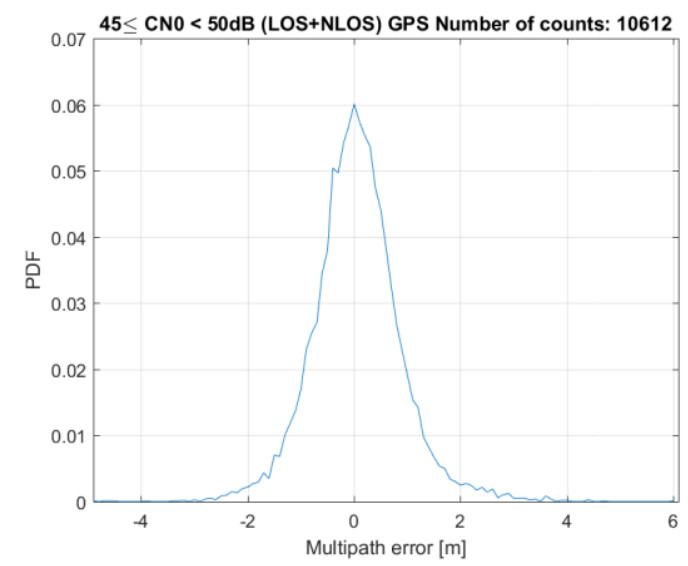

Figure 5 - Pdf of multipath error component in the 45-50 dB-Hz $C \backslash N_{0}$ band. GPS constellation case. $\mu=0.06[\mathrm{~m}], \sigma=0.83[\mathrm{~m}]$

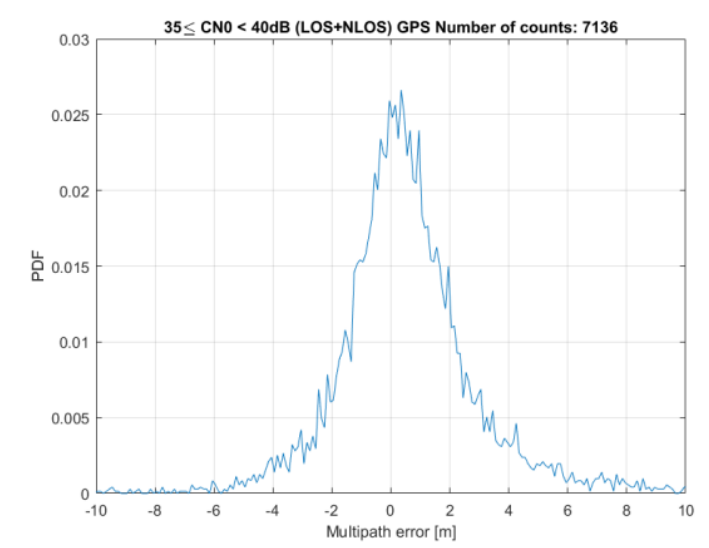

Figure 6 - Pdf of multipath error component in the $35-40 \mathrm{~dB}-\mathrm{Hz} C \backslash N_{0}$ band. GPS constellation case. $\mu=0.91[\mathrm{~m}], \sigma=5.18[\mathrm{~m}]$ 


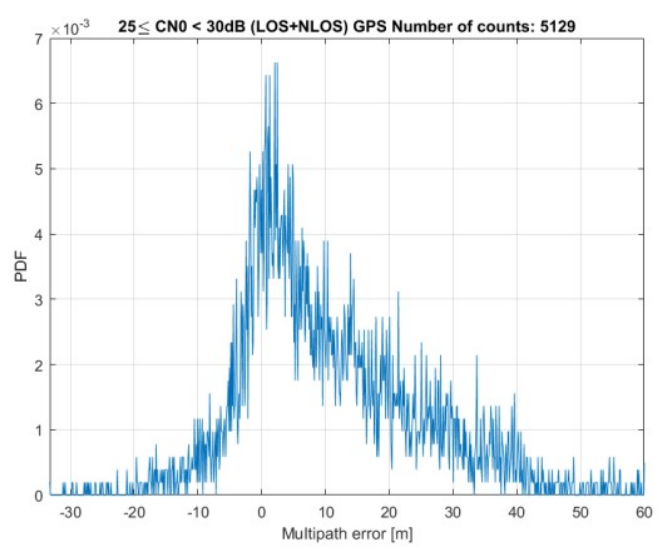

Figure 7-Pdf of multipath error component in the 25-30 dB-Hz $C \backslash N_{0}$ band. GPS constellation case. $\mu=11.48[\mathrm{~m}], \sigma=16.7[\mathrm{~m}]$

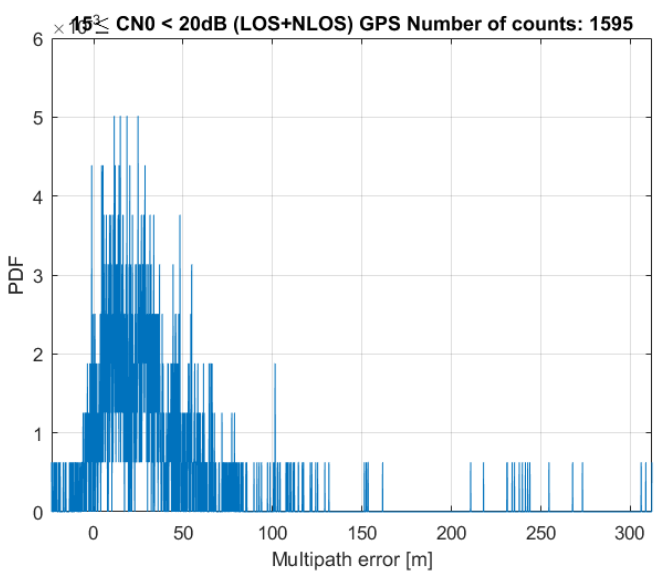

Figure 8 - Pdf of multipath error component in the

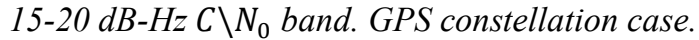
$\mu=29.8[\mathrm{~m}], \sigma=33.8[\mathrm{~m}]$.

\subsection{Multipath error characterization with respect to elevation angle classification}

The multipath characterization results obtained from the GPS constellation pseudorange measurements are presented next. Some of the results are commented first; then, the whole multipath error model is presented.

Figure 9Erreur! Source du renvoi introuvable. corresponds to the multipath error component pdf in the $70-80^{\circ}$ elevation angle range. The multipath error component has a symmetric Gaussian shape centred in 0 and a standard deviation of 0.82 meters. The pdf symmetry implies that the multipath error is mainly generated from LOS signals measurements as stated in section 5.3. We get similar results for the multipath error in Figure 5, 45-50 dB-Hz $\mathrm{C} \backslash \mathrm{N}_{0}$ range,. A connection between the two results could be seen: higher $\mathrm{C} / \mathrm{N}_{0}$ are usually related to the satellites with higher elevation angle. In this case, the multipath error component has a very low impact on the pseudorange measurement

Figure 10Erreur ! Source du renvoi introuvable. corresponds to the multipath error component pdf in the in the $30-40^{\circ}$ elevation angle range. As seen in Figure 6, section 5.4, the pdf starts to have a NonGaussian shape. The lower elevation angle could introduce some obstacles in the satellite-receiver line-of-sight. It can be seen that the pdf's values corresponding to the positive multipath errors seem to be higher than the negative part, in fact the mean value is equal to 2.45 meters. This phenomenon is probably due to the presence of NLOS conditions that result in positive biases as stated in section 5.3 .

Figure 11Erreur ! Source du renvoi introuvable. corresponds to the multipath error component pdf in the in the $10-20^{\circ}$ elevation angle range. In this picture, the probability density function is mostly positive biased; the pdf mean is equal to 12.9 meters. As well as before, the pdf positive error part is higher than the negative error part. The multipath error component pdf at this elevation angle seems thus to be generated manly by NLOS satellites. This result is expected since in an urban environment, the receiver-satellite line-of-sight is most of the time obstructed by obstacles, e.g. buildings.

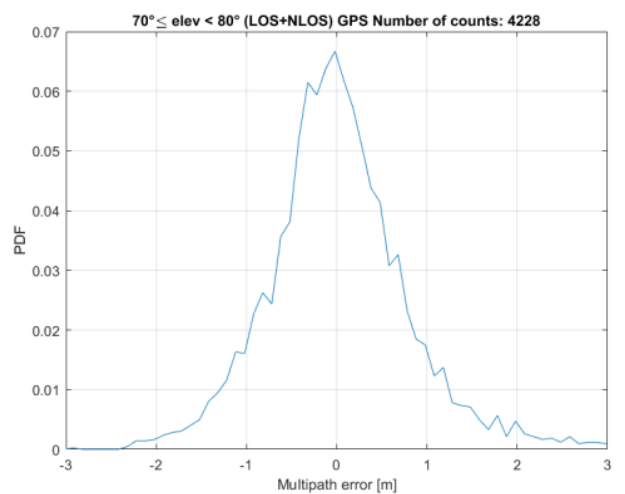

Figure 9-Pdf of multipath error component in the 70-80 elevation angle band. GPS constellation case. $\mu=0.012[\mathrm{~m}], \sigma=0.82[\mathrm{~m}]$. 


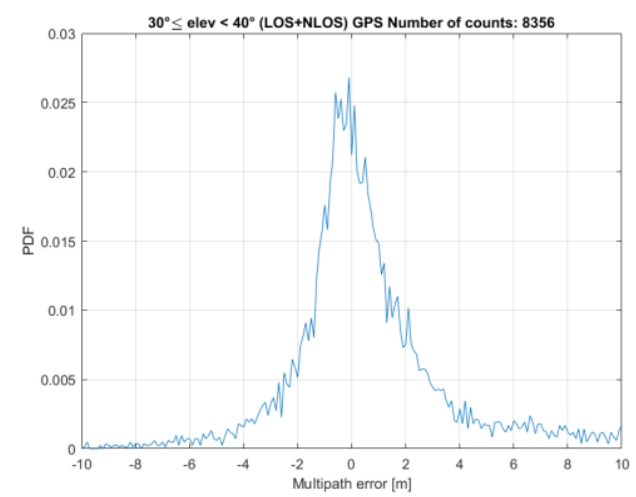

Figure 10- Pdf of multipath error component in the $30-40^{\circ}$ elevation angle band. GPS constellation case. $\mu=2.45[\mathrm{~m}], \sigma=8.9[\mathrm{~m}]$.

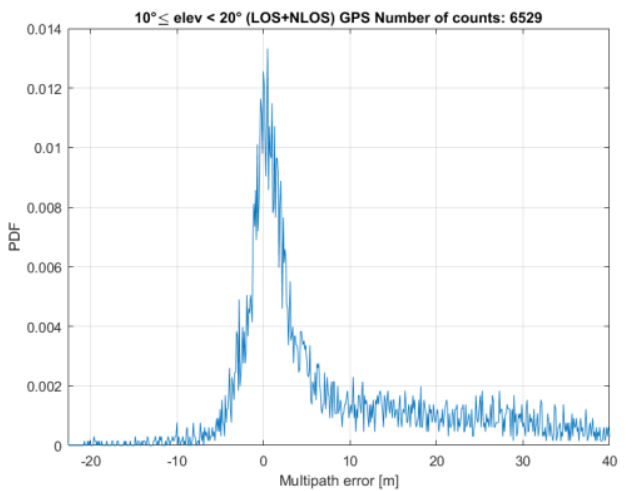

Figure 11- Pdf of multipath error component in the $10-20^{\circ}$ elevation angle band. GPS constellation case. $\quad \mu=12.9[\mathrm{~m}], \sigma=$ $23.67[\mathrm{~m}]$.

Table 2Erreur! Source du renvoi introuvable. contains the pdf's mean, standard deviation and the main peak's value for each different pdf characterized by a different elevation angle range. From this table it can be observed that from range $40-50^{\circ}$ to lower elevation angle values, the main peak beings to have a significant difference with the mean value. Moreover, the mean value is no longer around 0 . Therefore, it can be observed that from the $40-50^{\circ}$ range, a significant number of NLOS signals are received.

\begin{tabular}{|c|c|c|c|}
\hline $\begin{array}{c}\text { Elevation } \\
\text { angle } \\
\text { range } \\
\text { (degrees) }\end{array}$ & $\begin{array}{c}\text { Main } \\
\text { peak } \\
(\mathbf{m})\end{array}$ & $\begin{array}{c}\text { Mean } \\
(\mathbf{m})\end{array}$ & $\begin{array}{c}\text { Standard } \\
\text { deviation } \\
\mathbf{( m )}\end{array}$ \\
\hline $0-10$ & 0.2025 & 17.7762 & 31.6266 \\
\hline $10-20$ & 0.4517 & 12.8943 & 23.6727 \\
\hline $20-30$ & -0.1519 & 7.1971 & 13.8671 \\
\hline $30-40$ & -0.1001 & 2.4583 & 8.9342 \\
\hline $40-50$ & 0.3553 & 2.1349 & 7.9327 \\
\hline $50-60$ & 0.0986 & 0.7229 & 4.1832 \\
\hline $60-70$ & 0.1906 & 0.2042 & 1.5692 \\
\hline
\end{tabular}

\begin{tabular}{|c|c|c|c|}
\hline $70-80$ & -0.0199 & 0.0122 & 0.8219 \\
\hline $80-90$ & 0.1180 & -0.2726 & 0.8394 \\
\hline
\end{tabular}

characteristics per different elevation angle range

\subsection{Multipath error model: elevation angle vs. $C / N_{0}$}

In this section, a comparison between the capacity to recognise LOS and LOS satellites between the two multipath error model classifications, $\boldsymbol{C} / \boldsymbol{N}_{\mathbf{0}}$ and satellite elevation angle, is provided.

Let us take into account the two different tables provided in the previous sections, Table 1 and Table 2 , containing respectively the multipath error $\mathrm{pdf}$ statistics characterized by the received signal $C / N_{0}$ and the elevation angle.

From the comparison of these two tables, it can be observed that the multipath error component mean values are higher for low $\boldsymbol{C} / \boldsymbol{N}_{\mathbf{0}}$ values than they are for low satellite elevation angle values. This means that the $\boldsymbol{C} / \boldsymbol{N}_{\mathbf{0}}$ parameter allows for a better distinction between LOS and NLOS received signal conditions than the satellite elevation angle parameter: a higher number of NLOS satellites are found having a low $C / N_{0}$ value than having a low satellite elevation angle. .

To better understand this behaviour, in Figure 12 Satellite $\boldsymbol{C} / \boldsymbol{N}_{\mathbf{0}}$ in function of the elevation angles. the satellite $C / N_{0}$ as a function to the satellite elevation angle during the data campaign is provided. From this figure, it can be observed that for low elevation angle values, the $C / N_{0}$ has a large variation which goes from $10 \mathrm{~dB}-\mathrm{Hz}$ to $45 \mathrm{~dB}-\mathrm{Hz}$ (vertical axis): a lot of possible $C / N_{0}$ situations/received signal conditions, even quite high $C / N_{0}$ values, are allowed. On the contrary, for low $C / N_{0}$ values, the elevation angle values are less spread and more concentrated in the low values (horizontal axis): only a reduced number of situations/received signal conditions are allowed. 


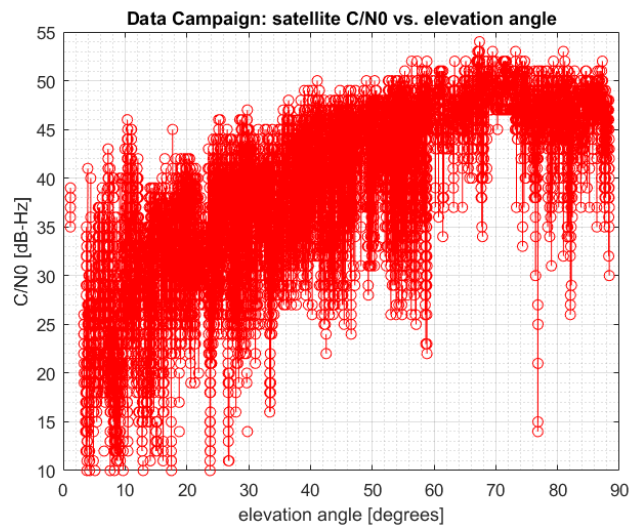

Figure 12 - Satellite $\mathrm{C} / \boldsymbol{N}_{\mathbf{0}}$ in function of the elevation angles.

\section{CONCLUSIONS AND FUTURE WORK}

In this paper, a pseudorange multipath error component isolation method is proposed and applied to the data collection of a measurement campaign in Toulouse urban area. The data campaign was conducted with a mass-market receiver in a moving vehicle.

The proposed method does not differentiate between multipath error and thermal noise components; this means that the proposed method and the obtained results are in fact a joint estimate of both components in only one term.

The isolated multipath error component has then been characterized by the calculation of its probability density function. Prior to the characterization process, the isolated multipath error component values are classified depending on the received signal $\mathrm{C} / \mathrm{N}_{0}$ and the satellite elevation angle.

This characterization has shown the impact of NLOS received signal conditions in the GNSS pseudorange measurements and on the multipath error component modeling: the multipath error component and thus, the pseudorange measurements, do not always follow a Gaussian distribution. Therefore, simply assuming that, in urban environments, GNSS measurements are Gaussian distribution can lead to strongly erroneous estimated position and/or a lack of integrity of the calculated position. In fact, for low $C / N_{0}$ values and for low satellite elevation angles, the presence of NLOS signals is quite important and the obtained distribution is non-Gaussian positive biased.

Finally, the $C / N_{0}$ received signal parameter allows for a better classification of the multipath error component received signal conditions: LOS or NLOS situations.
Future work will consist in:

- Characterize Multipath error model from Doppler measurements.

- Including Galileo and GLONASS in the measurement quality assessment.

- Taking advantage of the knowledge of the measurement pdf to optimize the navigation filter.

- Using an upward-looking camera and specific image-processing software to separate the satellite signals received in LOS and NLOS conditions; the classification will be made by detecting which satellites are directly visible or hidden behind an obstacle.

\section{References}

[1] D. J. R. Van Nee, "Multipath effects on GPS code phase measurements," NAVIGATION, Journal of Navigation, vol. 39, no. 2, 1992.

[2] J. M. Kelly, M. S. Braasch, and M. F. DiBenedetto, "Characterization of the effects of high multipath phase rates in GPS," GPS Solutions, vol. 7, no. 1, pp. 5-15, May 2003.

[3] B. M. Hannah, "Modelling and simulation of GPS multipath propagation," Ph.D. dissertation, Queensland University of Technology, Queensland, Australia, 2001.

[4] G. Moura and J. Castets, "Validation of deterministic simu- lation of GNSS reception in urban areas by comparison with measurement campaign," in Proc. of the European Navigation Conference GNSS, Toulouse, France, Apr. 2008.

[5] L. Hagerman, "Effects of multipath on coherent and non- coherent ranging receivers," The Aerospace Corporation, Tech. Rep. TOR-0073 (3020-03)-3, May 1973.

[6] E. Kaplan, Understanding GPS: Principles and Applications. Artech House Publishers, Feb. 1996.

[7] J. Farrell and T. Givargis, "Differential GPS reference station algorithm - design and analysis," IEEE Trans. Control Syst. Technol., vol. 8, no. 3, pp. 519-531, 2000.

[8] P. Brocard, "Integrity monitoring for mobile users in urban environment," 2016.

[9] Olivier Le Marchand, Philippe Bonnifait, Javier Ibanez-Guzman, David Betaille, Francois Peyret. Characterization of GPS multipath for passenger vehicles across urban environments. ATTI dell'Istituto Italiano di Navigazione, 2009, pp.77 88. <hal-00445114> 
